\title{
On the electronic structure and equation of state in high pressure studies of solids
}

\author{
B K GODWAL*, P MODAK and R S RAO \\ High Pressure Physics Division, Bhabha Atomic Research Centre, Mumbai 400 085, India
}

\begin{abstract}
We discuss the high pressure behaviour of zinc as an interesting example of controversy, and of extensive interplay between theory and experiment. We present its room temperature electronic structure calculations to study the temperature effect on the occurrence of its controversial axial ratio (c/a) anomaly under pressure, and the related electronic topological transition (ETT). We have employed a dense $63 \times 63 \times 29 \mathrm{k}$ point sampling of the Brillouin zone and find that the small $(c / a)$ anomaly near $10 \mathrm{GPa}$ pressure persists at room temperature. A weak signature of the anomaly can be seen in the pressure-volume curve, which gets enhanced in the universal equation of state, along with that of $K$-point ETTs. We attribute the change of slope in the universal equation of state near $10 \mathrm{GPa}$ pressure, mainly to hybridization effects. The temperature effect in fact enhances the possibility of $L$-point ETT. We find that the $L$-point ETT is very sensitive to exchange correlation terms, and hence we suggest that further refinements in the theoretical techniques are needed to resolve the controversies on the ETT in Zn.
\end{abstract}

Keywords. Electronic topological transition; electronic structure; c/a anomaly; high pressure; Zn.

\section{Introduction}

First principles electronic structure calculations have contributed significantly to high pressure studies, especially of solids. Along with $a b$ initio molecular dynamics (MD) simulations, these starting from the scratch calculations have been going through rapid expansion during the last two decades. Main merit of these first principles calculations is their predictive power, as they demand little a priori experimental data. A few well-established achievements of their independent predictive power are: (i) prediction of phase transition, even under physical conditions, which are not yet easily attainable in the laboratories (Godwal 1995); (ii) interpretation of mechanism of physical processes, especially in the case of observed anomalies by providing finer details of the calculations (Rao et al 1992, 2001a; Godwal et al 1998) and (iii) resolving the controversies, often with hints about the associated reasoning for the inappropriate interpretation (Sikka et al 1992; Godwal et al 1997).

The recent developments in experimental high pressure techniques, especially the tunable high pressure generation in the diamond anvil cell (DAC) along with laser heated high temperature conditions, have provided unique opportunity for the theorists to compare their predictions directly with experimental findings. With $50 \%$ volume compression routinely achieved in these experiments, significant changes are expected, and indeed found, in the electronic states, bonding characteristics, and atomic packing of condensed matter.

*Author for correspondence
Possibility of direct comparison between accurate high pressure experimental data and first principles theoretical predictions, has benefitted both these branches of investigations. Already, this kind of intense interaction between theory and experiment (Godwal 1995) has led to the development of various prescriptions for the exchangecorrelation terms in the density functional theories (Hohenberg and Kohn 1964), including the current generalized gradient approximation (GGA) and meta-GGA (Perdew et al 1996, 1999). Accuracy in state-of-the-art first principles electronic structure calculations is mainly limited by the approximations made for exchangecorrelation contribution to the total energy. Experience has shown that the limitation may lead to uncertainty in the absolute value of the computed quantity; but its variation with pressure is orders of magnitude more accurate, mainly due to mutual cancellation of errors.

In this presentation, we discuss the controversial high pressure behaviour of zinc as an interesting example of extensive interplay between theory and experiment.

\section{Zinc at high pressure: conflicting claims and explanations}

$\mathrm{Zn}$ has been extensively studied both theoretically and experimentally, for its high pressure behaviour for more than a decade now, with leading theoretical and experimental groups still at loggerheads with each other's conclusions (Lynch and Drickamer 1965; Daniuk et al 1989; Potzel et al 1989, 1995; Schulte et al 1991; Meenakshi et al 1992; Takemura 1995, 1997, 1999; Morgan et al 1996; Fast et al 1997; Godwal et al 1997; Novikov et al 1997, 
1999; Klötz et al 1998; Overhouser 1998; Olijnyk et al 2000; Li and Tse 2000; Rao et al 2001b; Kechin 2001; Steinle-Neumann et al 2001). It is a unique elemental solid for which 110,592 points in Brillouin zone (BZ) (with 2 atoms per primitive cell) were used for accurate integration (Steinle-Neumann et al 2001). It also has the distinction of forcing some of the investigators to contradict their own earlier experimental/theoretical inferences, though with understandable reasoning (Takemura 1995, 1997, 1999; Novikov et al 1997, 1999). As will be seen in our discussion, explanation of high pressure phenomena in $\mathrm{Zn}$ demands further improvements in the theoretical methods. It also reflects the current accuracy demands in some of the first principles calculations.

$\mathrm{Zn}$ crystallizes in the hexagonal close packed ( $h c p)$ structure under ambient conditions, and its axial ratio is large $(c / a=1.856)$ compared to the ideal close-packing value of 1.633. The large deviation from the close packing leads to anisotropy in the Fermi surface topology, and thus in transport and other physical properties. The application of pressure reduces $(c / a)$, and thus anisotropy. Any departure from the smooth decrease of $(c / a)$ under compression is termed as the '(c/a) anomaly'. A highly debated controversy in $\mathrm{Zn}$, persisting for a decade, is whether any such anomaly exists near $10 \mathrm{GPa}$ pressure. Moreover, Zn exhibits giant Kohn anomaly (GKA) (Kohn 1959; Kagan et al 1983) at $L$-point of the BZ at ambient pressure (Potzel et al 1995; Kechin 2001) (see figure 1). Another controversy in Zn (Potzel et al 1989, 1995; Morgan et al 1996; Novikov et al 1997; Klötz et al 1998; Olijnyk et al 2000; $\mathrm{Li}$ and Tse 2000; Rao et al 2001b; Kechin 2001) is about the destruction of GKA under pressure due to electronic topological transition (ETT) (Lifshitz 1960; Dagens 1978). As is well known, ETT refers to the shift of the electronic band extremum through the Fermi level $\left(E_{\mathrm{F}}\right)$, and the additional controversy is whether it is the cause of $(c / a)$ anomaly (Godwal

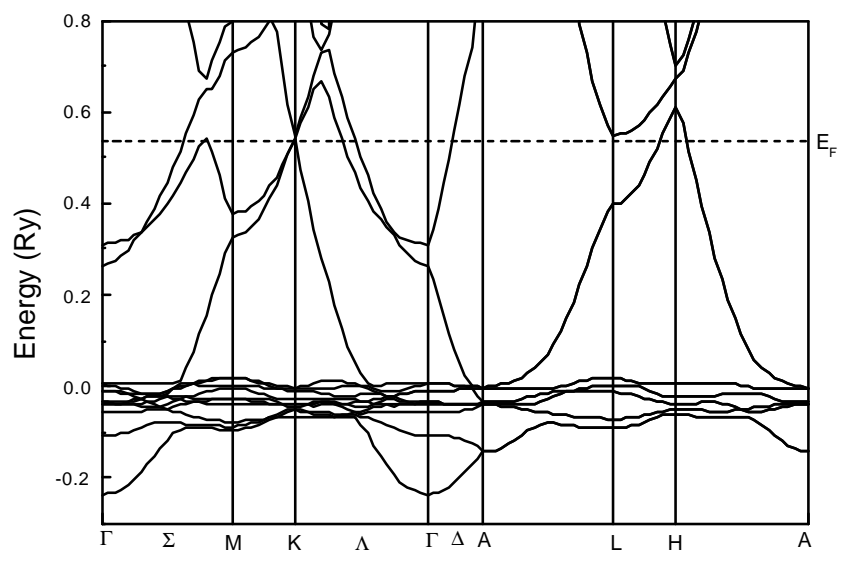

Figure 1. Electron energy band structure of $\mathrm{Zn}$ at normal pressure. et al 1997; Novikov et al 1997, 1999; Takemura 1999; Olijnyk et al 2000; Li and Tse 2000; Kechin 2001; Steinle-Neumann et al 2001).

The first theoretical prediction of the anomaly in the variation of $(c / a)$ as a function of pressure was by Meenakshi et al (1992) who attributed it to the shift of a van-Hove peak in the density of states through $E_{\mathrm{F}}$. Their studies helped to resolve the controversy on the disagreement between the experimental data of two groups (Lynch and Drickamer 1965; Schulte et al 1991), with subsequent experimental and theoretical investigations supporting their conclusions (Potzel et al 1995; Takemura 1995, 1997). Prominent among them were the direct observation of the $(c / a)$ anomaly in angle dispersive X-ray diffraction (ADXRD) data (Takemura 1995, 1997), and observation of anomaly in Lamb-Mössbauer factor in the high pressure Mössbauer spectroscopy, supported by the linear augmented plane wave (LAPW) calculations showing ETT at the $L$-point of the (BZ) at about the same compression (Potzel et al 1995). But the ADXRD data showed the anomaly at $(c / a)=\sqrt{ } 3$ which led to the suggestion that the high degeneracy of the reciprocal lattice vectors at this particular axial ratio might be responsible for the anomaly (Takemura 1995, 1997).

On the similar metal Cd, Godwal et al (1997) predicted two anomalies in its $(c / a)$ variation under compression; with the first at $V / V_{0}=0.95$ occurring due to ETT at the $K$-point with $c / a$ value different from $\sqrt{3}$, and the second corresponding to $V / V_{0}=0 \cdot 85$. These predictions were later supported by experiments (Takemura 1995, 1997) and other theoretical calculations (Novikov et al 1997).

Among the conflicting reports, Morgan et al (1996) observed rapid increase of mode Grüneisen parameter in the transverse acoustic branch $\left(\Sigma_{3}\right)$ above $6 \mathrm{GPa}$, and attributed it to the collapse of giant Kohn anomaly via ETT. However, subsequent studies by Klötz et al (1998) found regular behaviour. Also, the high pressure X-ray diffraction (XRD) experiments of Takemura (1999) at room temperature with helium as the pressure transmitting medium contradicted his earlier findings and revealed that there was no (c/a) anomaly, thus adding chaos into the confusion.

Many theoretical investigations supported the existence of (c/a) anomaly under compression in $\mathrm{Zn}$ (Meenakshi et al 1992; Fast et al 1997; Li and Tse 2000; Kechin 2001; Novikov et al 1997, 1999), though not much attention is being paid to reports of large anomalies (Lynch and Drickamer 1965; Fast et al 1997). But correlation of the anomaly to ETT has been debated and uncertainties still exist in this regard. Some of the phenomena attributed to the anomaly are, $K$-point ETT (Fast et al 1997), L-point ETT (Meenakshi et al 1992; Potzel et al 1995), combined influence of $K$ - and $L$-ETTs (Novikov et al 1999), and domains of different $c / a$ values (Novikov et al 1997). However, recent calculations 
(Steinle-Neumann et al 2001) blame the accuracy in the earlier computations due to poor $\mathbf{k}$-sampling and claims that the anomaly does not exist.

Briefly the frantic efforts on $\mathrm{Zn}$ at high pressure appeared to have resolved the issue of $(c / a)$ anomaly and ETT around the turn of the century, with the general understanding that they could exist at low temperature. Thus the observed anomaly in the Lamb-Mössbauer factor at 6.6 GPa and $4 \mathrm{~K}$ (Potzel et al 1995) indicated drastic softening of low frequency acoustic or optical phonons and was interpreted as the effect of destruction of giant Kohn anomaly driven by ETT. Temperature was supposed to play a crucial role in wiping out the experimental observation at room temperature of the signatures of ETT (Klötz et al 1998) though Morgan's experiments (Morgan 1996) showed otherwise. As is well known, the temperature smearing of the Fermi distribution function in the rigid band picture would reduce the ETT effects in the physical properties.

Further verification by experimentalists and theorists continued, rekindled by the observation of anomalous broadening of Raman line width near $10 \mathrm{GPa}$ pressure in the room temperature measurements of Olijnyk et al (2000). Among the recent investigations, Overhauser suggested a first-order transition to a commensurate spin density-wave (SDW) (Overhauser 1998) to explain the apparently contrasting behaviour in Lamb-Mössbauer factor (Potzel et al 1995) and phonon frequencies (Klotz et al 1998) under pressure. Later, $\mathrm{Li}$ and Tse (2000) obtained the phonon frequencies and the mode Grüneisen parameters $(\gamma)$, which showed significant softening of an acoustic mode near $10 \mathrm{GPa}$. They thus explained the $(c / a)$ anomaly due to the softening of phonon modes as it affects the compressibility. $\mathrm{Li}$ and Tse, and Kechin (2001) supported the occurrence of ETT and the anomaly. Though no cla anomaly was obtained in the calculations of Steinle-Neumann et al (2001), they had neglected the spin-orbit interaction, and according to Kechin (2001), it could affect the anisotropy of compressibility and thus (c/a) anomaly, because of two different types of Fermi surface sheets arising out of ETT. Also the conclusions of Steinle-Neumann et al (2001) were in contrast to those of $\mathrm{Li}$ and Tse who also employed comparable $\mathbf{k}$ sampling. Thus there is no consensus yet on the high pressure behaviour of $\mathrm{Zn}$.

In view of the role of temperature factor mentioned above, we report here the effect of temperature on the electronic structure, and thus on the axial ratio anomaly, and ETT in $\mathrm{Zn}$. We have carried out accurate room temperature electronic structure calculations on $\mathrm{Zn}$ with generalized gradient approximation (GGA), based on the LAPW method. We have modified the full potential electronic structure code so that the density distribution of electrons in the self-consistent cycle changes in accordance with finite temperature occupation of electron energy levels. Our results show a small (c/a) anomaly at room temperature, comparable to that at $0 \mathrm{~K}$. Though our calculations do not show any ETT at $L$-point, we comment upon the feasibility of its occurrence, in view of its sensitivity to exchange-correlation terms, and suggest about further investigations.

We give a brief description of our calculations in the next $\S$ and present the results and discussion in $\S 4$, followed by conclusions in $\S 5$.

\section{Details of calculations}

Our room temperature calculations were based on the generalization of Hohenberg-Kohn (1964) theorem to non-zero temperature (Mermin 1965; Gupta and Rajagopal 1982; Kohn and Vashishta 1983). The main modification needed from the $0 \mathrm{~K}$ formulation was in evaluating the charge density with the Fermi function, as hardly any modification was needed in exchange correlation terms at $300 \mathrm{~K}$ (Gupta and Rajagopal 1982). We accordingly modified the tetrahedron method (Lehmann and Taut 1972) of BZ integration in evaluating the charge density. The associated weights with the tetrahedral corners for BZ integration were evaluated following Blöchl et al (1994). For further details see Godwal et al (2002a). The necessary entropy $(S)$ contribution due to electron distribution function, $f$, at temperature, $T$, is given by

$$
-S T=-k_{\mathrm{B}} \sum_{i}\left[f_{i} \ln \left(f_{i}\right)+\left(1-f_{i}\right) \ln \left(1-f_{i}\right)\right] T .
$$

where $f_{i}(\varepsilon)=1 /\left[\exp \left\{(\varepsilon-\mu) / k_{\mathrm{B}} T\right\}+1\right]$, is the Fermi distribution function with $\varepsilon$ denoting the eigenvalue of the $i$ th quantum state, whereas $\mu$ and $k_{\mathrm{B}}$ denote the chemical potential and Boltzman constant, respectively. For $\mathrm{Zn}$ at room temperature, the entropy contribution to free energy is less than a $\mu \mathrm{Ry}$.

In our calculations, the $3 d^{10} 4 s^{2}$ states of $\mathrm{Zn}$ formed the valence part, treated semi-relativistically with spin-orbit effect as perturbation, whereas the rest of the occupied electron orbitals constituted the ion core and were treated fully relativistically. The GGA was employed for the exchange-correlation terms as per the prescriptions of Perdew et al (1996). We adapted the FP-LAPW computer code WIEN97 of Blaha et al (1988, 1997). About 280 plane waves were found adequate to expand the valence electron orbitals in the interstitial region $\left(R K_{\max }=9\right.$ in WIEN97). We employed the $63 \times 63 \times 29$ mesh in the BZ for $\mathbf{k}$-space integration, which is a better and more uniform sampling than employed by SteinleNeumann et al (2001). The calculations were carried out for $0 \mathrm{~K}$ as well as for room temperature. The $(c / a)$ ratios at each volume compression were varied to estimate the optimum value that minimized the free energy, thus enabling us to obtain the (c/a) variation under compression. The calculations were often repeated with LDA (Perdew and Wang 1992) for comparison. 


\section{Results and discussion}

Figure 1 shows the electron band structure of $\mathrm{Zn}$ at ambient condition computed with GGA. Reliability of our room temperature calculations can be judged by a few estimates of ambient pressure physical quantities, like equilibrium volume of 203.4 (a.u.) $)^{3}$ per unit cell, $c / a$ ratio of 1.875 , and bulk modulus of $58 \mathrm{GPa}$, compared to the experimental values of 205.25 (a.u. $)^{3}, 1.856$, and $56.5 \mathrm{GPa}$, respectively (Morgan et al 1996). As mentioned in $\S 1$, discrepancies of such relative magnitudes are common in the first principles calculations.

The calculated total energy vs $(c / a)$ for several reduced volumes $\left(V / V_{0}, V_{0}\right.$ being the volume at ambient pressure) are shown in figure 2, which show somewhat broad minima. The systematically calculated $(c / a)$ values from these curves show some anomaly near $V / V_{0}=0.90$, with the deviation of about 0.006 from the smooth decrease (see figure 3).

The small deviation in the value of $(c / a)$ from the smooth variation agrees well with that obtained by $\mathrm{Li}$ and Tse (2000). Thus, identification of this anomaly in the Xray diffraction experiments with the currently attainable accuracy may be difficult. The pressure versus volume $(P-V)$ curve obtained by calculating volume derivatives of total energies also shows a little deviation from smooth

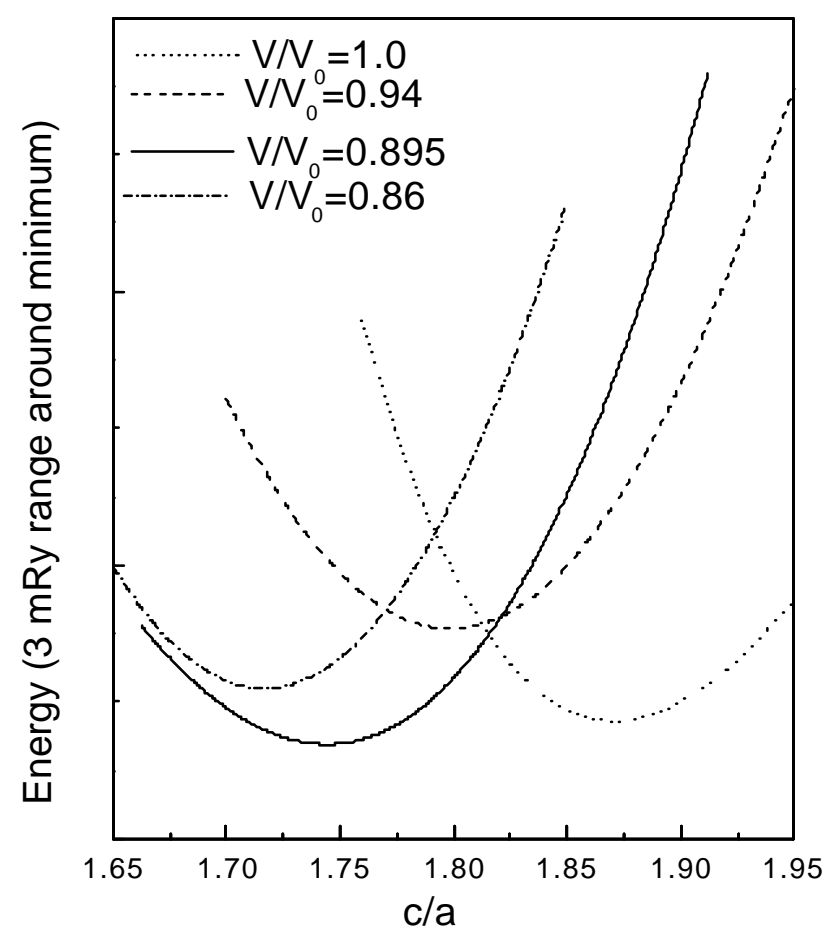

Figure 2. The total energies at various volumes as a function of axial ratio $(c / a)$. The energy scales are shifted for different $\left(V / V_{0}\right)$ so that $3 \mathrm{mRy}$ range near the minimum energies are depicted. Thus minimum energy positions for different compressions are not systematically related to one another. variation (figure 4) at the same volume compression (near $10 \mathrm{GPa}$ pressure). Note that the curve shown in figure 4 does not include the lattice thermal contribution to pressure, and temperature effect on the electronic structure only is considered here. The lattice thermal contribution can be evaluated following Godwal and

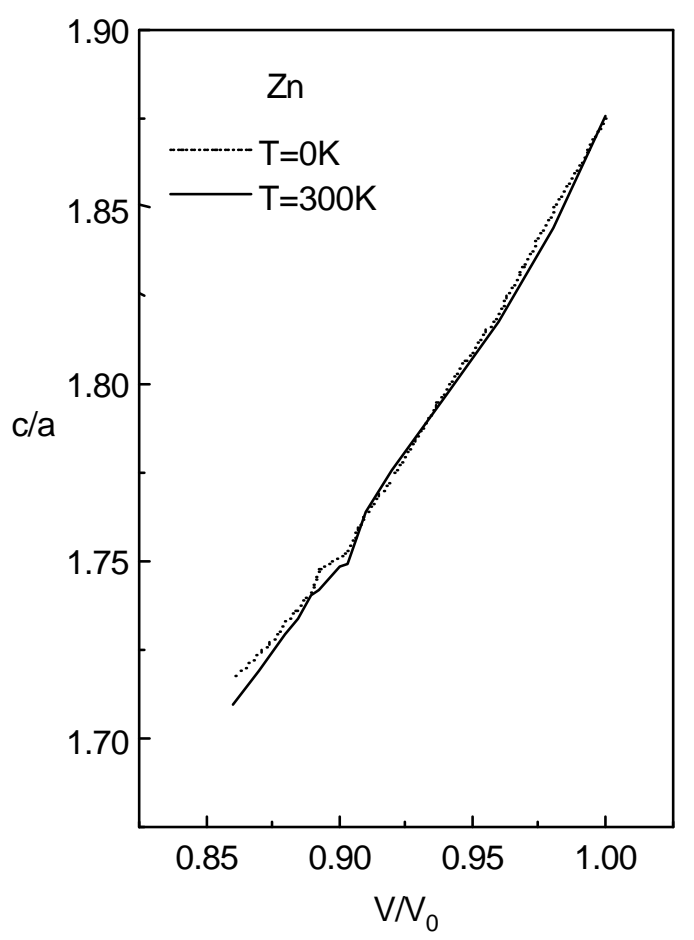

Figure 3. Room temperature (c/a) variation with volume compression in $\mathrm{Zn}$, obtained by electronic structure calculations. The (dashed) curve of $0 \mathrm{~K}$ results are also shown for comparison.

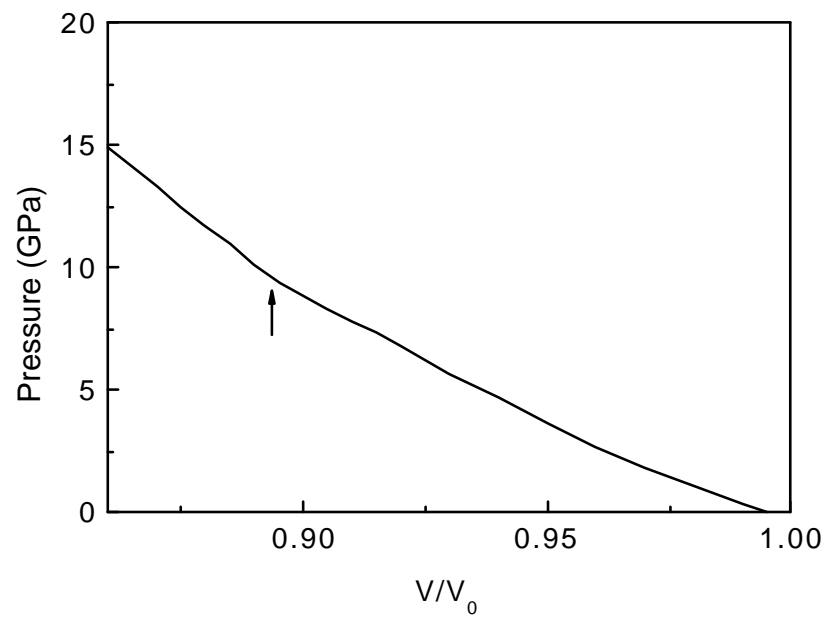

Figure 4. Equation of state obtained by $300 \mathrm{~K}$ electronic structure calculations. The arrow indicates the slight deviation from smooth increase of pressure with volume compression. 
Jeanloz (1989) using Grüneisen parameter, $\boldsymbol{\gamma}$ and have been reported elsewhere (Rao et al 2001a).

To obtain better signatures of any electronic transition, we transformed the equation of state in the universal form (UEOS) (Rose et al 1984). The UEOS is given by

$$
\ln H=\ln K_{0}+\eta(1-X),
$$

where $H=\left(P X^{2}\right) / 3(1-X)$ and $X=\left(V / V_{0}\right)^{1 / 3}, \eta$ being related to the pressure derivative $\left(K_{0}^{\prime}\right)$ of the bulk modulus, $K_{0}$ by

$$
\eta=3\left(K_{0}^{\prime}-1\right) / 2 \text {. }
$$

It is known that deviation from linearity in the UEOS suggests about the subtle electronic transitions, like $s$ to $d$ transition (Sikka 1988), and ETT (Godwal et al 2002b) in the absence of any other major cause like structural transition. The UEOS, shown in figure 5 clearly shows deviation from linearity near $V / V_{0}=0.96$ and 0.90 . The former correlates well with the $K$-point ETT, and the latter correspond to the compression at which the (c/a) anomaly occurs.

We have also investigated sensitivity of the electron energy eigen values near $E_{\mathrm{F}}$ at the $L$-point in the $\mathrm{BZ}$ on the exchange-correlation terms employed, as its position with respect to $E_{\mathrm{F}}$ is related to GKA and ETT (Meenakshi et al 1992; Potzel et al 1995; Kechin 2001) (see figure 1). Briefly, if the eigenvalue at the $L$-point, which is $15.5 \mathrm{mRy}$ higher in energy than Fermi energy $\left(E_{\mathrm{F}}\right)$ at ambient pressure (figure 1), descends through $E_{\mathrm{F}}$, the ETT would take place destroying the GKA. As already mentioned, there are conflicting claims about this transition by various investigators (Potzel et al 1995; Morgan et al 1996; Takemura 1997; Klötz et al 1998; Olijnyk et al 2000). The present calculations show that the eigenvalue never reaches $E_{\mathrm{F}}$ up to the highest compression

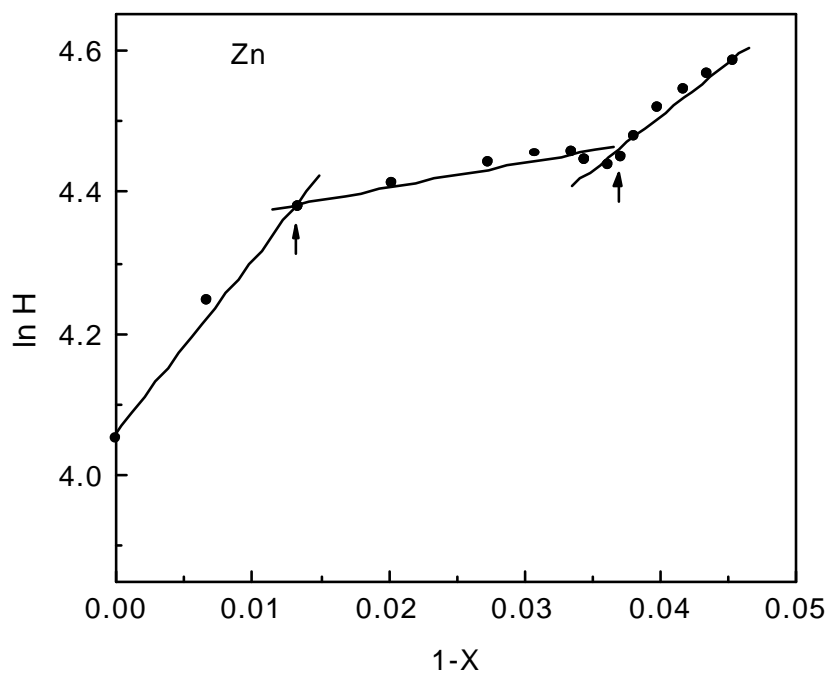

Figure 5. The universal equation of state of $\mathrm{Zn}$ based on electronic structure calculations. studied (i.e. $V / V_{0}=0 \cdot 8$ ). However, its closest position with respect to $E_{\mathrm{F}}$ is sensitive to the assumed form of the exchange-correlation terms. Briefly, the $L_{1}$ eigenvalue approaches within $6.5 \mathrm{mRy}$ ( $7 \mathrm{mRy}$ in $0 \mathrm{~K}$ calculations) with GGA, and $2.5 \mathrm{mRy}$ with local density approximation (Perdew and Wang 1992). Thus the sensitivity of the position of $L_{1}$ level (relative to $E_{\mathrm{F}}$ ) to exchange-correlation terms indicates that an appropriate prescription of these terms might as well bring the $L_{1}$ level below $E_{\mathrm{F}}$. Note that shifts involved in some of the GW (Green's function-screened Coulomb) calculations on metallic systems are by order of magnitude larger (Hybertsen and Louie 1985), than a few mRy shift needed for the ETT to occur at the $L$-point. As there are experimental indications of the occurrence of ETT from the Raman studies of Olijnyk et al (2000), the resistance measurements (Lynch and Drickamer 1965; Kechin 2001; Alka et al 2002), etc. (Potzel et al 1995; Morgan et al 1996), it would be interesting to see what the quasi-particle excitation bands, like those obtained by GW-type calculations (Hybertsen and Louie 1985) would inform about ETT in $\mathrm{Zn}$. If the ETT were to occur, the anomaly in axial ratio should be larger than that obtained in the present work. Also, temperature effects bring the $L_{1}$-level a bit closer to $E_{\mathrm{F}}$ (by $0.5 \mathrm{mRy}$ ), which along with the temperature smearing of the Fermi function would help the ETT to occur.

As the ETT at the $L$-point did not show up in our calculations, we looked for other features in the band structure, which could explain the change of slope seen in figure 5. Comparison of the band structure at various pressures showed that some of the unoccupied levels, like

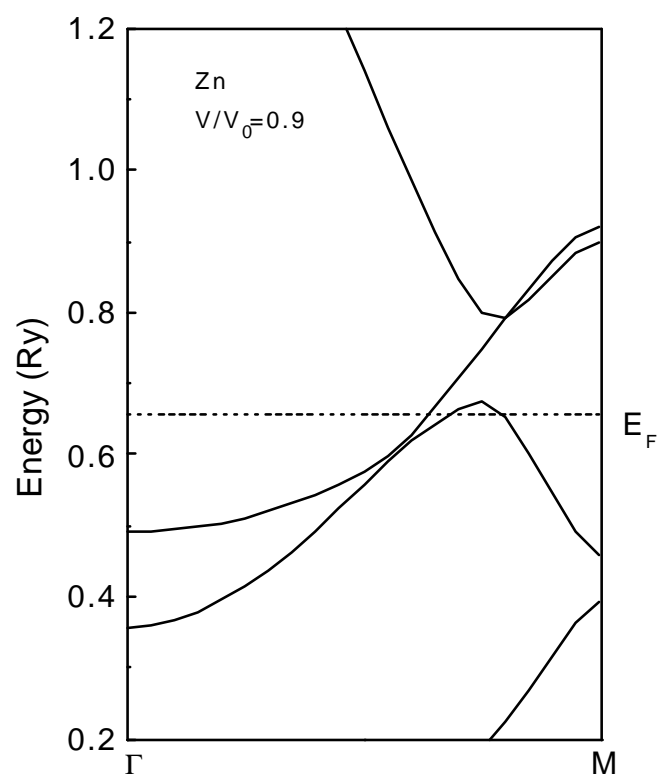

Figure 6. Electron energy band structure of $\mathrm{Zn}$ along $\Gamma M$ direction of $\mathrm{BZ}$ at volume compression $\left(V / V_{0}\right)=0.90$. 
$L_{1}$ level near $E_{\mathrm{F}}$, move towards $E_{\mathrm{F}}$ at low compressions; but around $10 \mathrm{GPa}$, the competing counter effect of $s-p$ hybridization dominates and shifts them again to higher energies. For example, we found that a hybridization gap in the $\Gamma M$ direction (figures 1 and 6 ) decreases up to $10 \mathrm{GPa}$, from its ambient pressure value of $132 \mathrm{mRy}$ to $116 \mathrm{mRy}$; then starts to increase, signaling the onset of dominance of hybridization repulsion over the shift of bands under compression. Thus the relative positions of some of the bands start to re-order above $10 \mathrm{GPa}$, leading to changes in the rate of change of compressibility, and thus the change in slope in UEOS (figure 5). As noted earlier, similar effects on the UEOS are known to occur due to band re-ordering leading to $s-d$ transition under pressure (Sikka 1988).

\section{Conclusions}

Thus our $300 \mathrm{~K}$ results show that temperature effects on the electronic structure do not hinder the occurrence of ETT and the $(c / a)$ anomaly in zinc at room temperature, which is contrary to the earlier expectations. The present calculations show that the band structure itself changes, due to modification of the electron density distribution, and brings the $L$-point eigenvalue closer to the Fermi level. This change along with temperature smearing of Fermi distribution function enhances the occurrence of ETT. We have also shown that the $L$-point ETT is very sensitive to the exchange-correlation terms employed. Thus the controversy in high pressure behaviour of $\mathrm{Zn}$ has yet not been resolved. It is our expectation, on the grounds of available experimental and theoretical evidence, that ETT at $L$-point occurs around $10 \mathrm{GPa}$ pressure. But state-of-the-art density functional theory based calculations with LDA or GGA for exchange-correlation cannot reproduce this ETT. The high pressure behaviour of $\mathrm{Zn}$ illustrates the constructive interplay between theory and experiment, and challenges the theorists to improve upon their methods. In the experimental side, some low temperature Fermi surface studies of $\mathrm{Zn}$ under pressure would also be useful to examine the occurrence of ETT at $L$-point.

\section{Acknowledgement}

We are grateful to $\mathrm{O} \mathrm{K}$ Andersen for a useful discussion, which led us to probe the band structure in more detail.

\section{References}

Alka B Garg et al 2002 J. Phys. Condens. Matter 148795 Blaha P, Schwarz K and Dederichs P H 1988 Phys. Rev. B37 2792
Blaha P, Schwarz K and Luitz J 1997 WIEN97, Vienna University of Technology (Improved and updated Unix version of the original copyrighted WIEN code, which was published by Blaha P, Schwarz K, Sorantin P and Trickey S B 1990 Comput. Phys. Commun. 59 399)

Blöchl P E, Jepsen O and Andersen O K 1994 Phys. Rev. B49 16223

Dagens L 1978 J. Phys. F Metal Phys. 82093

Daniuk S, Jarlborg T, Kontrym-Sznajd G, Majsnerowski J and Stachowiak H 1989 J. Phys. Condens. Matter 18397

Fast L, Ahuja R, Nordstrom L, Wills J M, Johansson B and Eriksson O 1997 Phys. Rev. Lett. 792301

Godwal B K 1995 Curr. Sci. 681087

Godwal B K and Jeanloz R 1989 Phys. Rev B40 7501

Godwal B K, Jayaraman A, Meenakshi S, Rao R S, Sikka S K and Vijayakumar V 1998 Phys. Rev. B57 773

Godwal B K et al 2002a Solid State Commun. (accepted)

Godwal B K et al 2002b Phys. Rev. B65 140101(R)

Godwal B K, Meenakshi S and Rao R S 1997 Phys. Rev. B56 14871

Gupta U and Rajagopal A K 1982 Phys. Rep. 87259

Hohenberg P C and Kohn W 1964 Phys. Rev. 136 B864

Hybertsen M S and Louie S G 1985 Phys. Rev. Lett. 551418

Kagan Yu, Pushkarev V V and Holas A 1983 Zh. Eksp. Teor. Fiz. 841494 [Sov. Phys. JETP 57 870]

Kechin V V 2001 Phys. Rev. B63 045119

Klöz S, Braden M and Besson J M 1998 Phys. Rev. Lett. 81 1239

Kohn W 1959 Phys. Rev. Lett. 2393

Kohn W and Vashishta P 1983 in Inhomogeneous electron gas (eds) $\mathrm{S}$ Lundquist and $\mathrm{NH}$ March (New York: Plenum) p. 124

Lehmann G and Taut M 1972 Phys. Status Solidi (b) 54469

Li Z and Tse J S 2000 Phys. Rev. Lett. 855130

Lifshitz I M 1960 J. Expt. Theor. Phys. 381569 [Sov. Phys. JETP 11 1130]

Lynch R W and Drickamer H G 1965 J. Phys. Chem. Solids 26 63

Meenakshi S, Vijayakumar V, Godwal B K and Sikka S K 1992 Phys. Rev. B46 14359

Mermin N D 1965 Phys. Rev. A137 1441

Morgan J G, Von Dreele R B, Wochner P and Shapiro S M 1996 Phys. Rev. B54 812

Novikov D L, Freeman A J, Christensen N E, Svane A and Rodriguez C O 1997 Phys. Rev. B56 7206

Novikov D L, Katsnelson M I, Trefilov A V, Freeman A J, Christensen N E, Svane A and Rodriguez C O 1999 Phys. Rev. B59 4557

Olijnyk H, Jephcoat A P, Novikov D L and Christensen N E 2000 Phys. Rev. B62 5508

Overhauser A W 1998 Phys. Rev. Lett. 814022

Perdew J P and Wang Y 1992 Phys. Rev. B45 13244

Perdew J P, Burke K and Ernzerhof M 1996 Phys. Rev. Lett. 77 3865

Perdew J P, Kurth S, Zupan A and Blaha P 1999 Phys. Rev. Lett. 822544

Potzel W, Adlassnig W, Moser J, Schafer C, Steiner M and Kalvius G M 1989 Phys. Rev. B39 8236

Potzel W, Steiner M, Karzel H, Schiessl W, Kofferlein M, Kalvius G M and Blaha P 1995 Phys. Rev. Lett. 741139 
Rao R S, Godwal B K and Sikka S K 1992 Phys. Rev. B46 5780

Rao R S, Modak P and Godwal B K 2001a in Advances in high pressure science and technology (eds) A K Bandyopadhyay, D Varandani and Lal Krishan (New Delhi: National Physical Laboratory) p. 206

Rao R S, Modak P and Godwal B K 2001b Phys. Rev. Lett. 87 259601

Rose J H, Smith J R, Guinea F and Ferrante J 1984 Phys. Rev. B29 2963
Schulte O, Nikolaenko A and Holzapfel W B 1991 High Pressure Res. 6169

Sikka S K 1988 Phys. Rev. B38 8463

Sikka S K, Godwal B K and Rao R S 1992 High Pressure Res. 10707

Steinle-Neumann G, Stixrude L and Cohen R E 2001 Phys. Rev. B63 054103

Takemura K 1995 Phys. Rev. Lett. 751807

Takemura K 1997 Phys. Rev. B56 5170

Takemura K 1999 Phys. Rev. B60 6171 